\title{
Médiévales
}

Langues, Textes, Histoire

65 | automne 2013

Le couple dans le monde franc

\section{Gianluca RACCAGNI, The Lombard League (1167-1225)}

Oxford/New York, Oxford University Press, 2010, 231 p.

\section{Sylvain Parent}

\section{Q OpenEdition}

1 Journals

Édition électronique

URL : https://journals.openedition.org/medievales/7156

DOI : $10.4000 /$ medievales. 7156

ISSN : $1777-5892$

Éditeur

Presses universitaires de Vincennes

Édition imprimée

Date de publication : 1 décembre 2013

Pagination : 205-208

ISBN : 978-2-84292-396-9

ISSN : 0751-2708

Référence électronique

Sylvain Parent, « Gianluca raccagnı, The Lombard League (1767-1225) », Médiévales [En ligne], 65 | automne 2013, mis en ligne le 20 janvier 2014, consulté le 24 avril 2022. URL : http://

journals.openedition.org/medievales/7156; DOI : https://doi.org/10.4000/medievales.7156

Ce document a été généré automatiquement le 24 avril 2022.

Tous droits réservés 


\title{
Gianluca RACCAGNI, The Lombard League (1167-1225) \\ Oxford/New York, Oxford University Press, 2010, 231 p.
}

\author{
Sylvain Parent
}

\section{RÉFÉRENCE}

Oxford/New York, Oxford University Press, 2010, 231 p.

1 G. Raccagni, qui enseigne à l'université d'Édimbourg, aborde dans ce livre un thème très classique - voire rebattu - de l'histoire italienne du XII siècle. Il souligne pourtant d'entrée de jeu une sorte de paradoxe: aucune synthèse actualisée n'était disponible sur le sujet (la dernière synthèse publiée en anglais date de 1866). La Ligue lombarde a en effet bénéficié d'une très grande fortune historiographique depuis le XVIII ${ }^{\mathrm{e}}$ siècle et a connu un véritable regain d'intérêt en Italie dans la seconde moitié du XIX ${ }^{\mathrm{e}}$ siècle, en particulier durant la période du Risorgimento : le thème est alors devenu très populaire et a été décliné de multiples manières, dans les romans, les opéras, la poésie ou encore la peinture. Si l'auteur revient rapidement sur ces récupérations diverses, politiques et artistiques, l'objectif de son travail est ailleurs: en reprenant les dossiers documentaires disponibles, il veut surtout étudier le fonctionnement de cette forme particulière d'association militaire, politique et diplomatique, que fut la Ligue lombarde. Pour donner plus de profondeur à sa démonstration, il choisit un arc chronologique plus vaste que dans les monographies précédentes, et poursuit au-delà de la paix de Constance de 1183, jusqu'en 1226, pour mieux montrer que cette association a survécu en temps de paix.

Le premier chapitre («Lombardy before the Lombard League») sert à planter le décor et revient sur les évolutions politiques de la Lombardie depuis le haut Moyen Âge et sur l'histoire tourmentée du royaume d'Italie depuis le démantèlement de l'empire carolingien jusqu'à l'apparition des premières communes entre la fin du XI siècle et le début du XII ${ }^{e}$ siècle. Dès cette époque, les communes naissantes ont pris l'habitude de 
former des ligues ou des coalitions. Au sein de ces réseaux d'alliances, l'attitude de Milan est de plus en plus hégémonique et tend à polariser les relations entre les communes de la région en groupes pro- et anti-milanais. Les divisions qui apparaissent alors entre ces cités lombardes ont joué un rôle important dans la vie de la future Ligue. La politique de Frédéric Barberousse, qui a organisé six expéditions en Italie entre 1152 et 1190 afin de restaurer son autorité, a été décisive dans la constitution de cette Ligue. Les villes partisanes de l'empereur ont alors reçu une plus grande autonomie, tandis que les villes ennemies comme Milan et ses alliés ont été strictement encadrées.

Une fois ces rappels généraux effectués, l'auteur en vient à la naissance et au développement de la Ligue jusqu'à la paix de Constance de 1183 («Formation, Expansion, and Membership of the Lombard League to $1183 »)$. Pour lui, le prélude de cette Ligue lombarde est à chercher dans la formation de la ligue de Vérone (1164-1166) : malheureusement, aucun document d'archive n'a survécu sur cette ligue de villes qui s'est rebellée contre Frédéric Barberousse, et qui compte notamment Venise, Vérone, Padoue et Vicence - son existence est uniquement rapportée dans les chroniques. C'est en 1167, lorsque l'empereur part vers le sud, que se forme une nouvelle alliance qui prendra le nom de Ligue lombarde. Cette nouvelle alliance entre des cités lombardes qui se faisaient jusqu'alors la guerre est aussi une alliance de paix, et Milan a ainsi dû renoncer à nombre de ses revendications. La question de la détermination d'une date de naissance officielle de cette Ligue lombarde est intéressante en ceci qu'elle a fait l'objet de récupérations: d'après la chronique de Bernardino Corio, la reconstruction de Milan a été planifiée au cours d'une assemblée qui s'est tenue dans le monastère de Pontida, près de Milan, le 7 avril 1167; à partir du $\mathrm{XIX}^{\mathrm{e}}$ siècle, c'est précisément cette assemblée qui a été vénérée comme le véritable début de la Ligue lombarde. D'ailleurs, certains groupuscules ou partis politiques d'extrême droite, comme la Ligue du Nord, continuent toujours de perpétuer cette tradition en se réunissant chaque année à cette date pour célébrer le début de la Ligue. En décembre 1167, la Ligue compte alors seize participants, et dès 1168 le nom officiel de Societas Lombardie commence à être utilisé. Le nombre des participants s'accroît après la fuite de Frédéric I $^{\mathrm{er}}$ en Allemagne, où il demeure de mars 1168 à 1174. G. Raccagni agrémente ce chapitre de plusieurs cartes très utiles pour mieux visualiser les fluctuations d'alliances et l'évolution de la composition de la Ligue à partir de 1167.

L'auteur entame ensuite une série de trois chapitres dont l'approche est plus thématique. Le chapitre 3 ( «Structure of the League ») fait le point sur la manière dont s'organise cette association. Si elle ne possède pas à proprement parler de quartier général, elle a cependant un ensemble de règles et un système de gouvernement propres, avec notamment des recteurs (rectores), choisis dans les cités alliées, qui représentent ses intérêts. L'appartenance à la Ligue engage les cités les unes envers les autres et celles-ci ne peuvent pas, à titre individuel, déclarer la guerre ou faire la paix sans son consentement. Elle a souvent été décrite comme une ennemie naturelle de la grande noblesse féodale; $G$. Raccagni nuance ce constat et rappelle que certains grands nobles l'ont rejointe, comme Obizzo Malaspina ou Ezzelino da Romano. Ce chapitre donne également à l'auteur l'occasion de revenir sur le problème épineux de la documentation. La Ligue n'ayant pas d'archives centralisées, l'historien doit naviguer dans une documentation peu abondante, éclatée entre les différentes cités et très inégalement conservée - pour Milan par exemple, qui a été le membre le plus important de la Ligue et son leader à partir de 1175 , on a perdu à peu près tous les documents. La seconde spécificité est que la plupart des documents ont survécu sous la 
forme de copies du XIII ${ }^{\mathrm{e}}$ siècle, notamment dans les libri iurium des communes. L'examen de ces traces documentaires montre cependant que des collections de documents existaient et circulaient en Italie du Nord.

Le chapitre 4 ("Activity of the League ») s'attarde sur les diverses facettes de son activité qui se manifeste tout d'abord dans le domaine de la guerre. G. Raccagni remarque que, si la Ligue a en général été décrite comme une alliance anti-impériale, ses implications dans les activités militaires ont été peu étudiées. Il faut néanmoins rappeler que les attestations d'une intervention armée de la Ligue sont ténues: l'une des principales preuves d'une implication directe des recteurs de la Ligue dans l'organisation d'une campagne militaire vient de 1174-1176, lorsqu'il faut venir en aide à Alessandria : c'est le seul moment où les sources parlent d'un exercitus Lombardorum (sans qu'on en sache davantage, cependant, sur l'organisation concrète de cette armée). Le deuxième versant de cette activité est l'aide réciproque entre membres, qui implique l'assistance diplomatique à la cour pontificale ou auprès de l'empereur. Les cités ne pouvaient pas déclarer la guerre ou signer une paix sans l'assentiment de la Ligue. Enfin, si la Ligue apporte aide et assistance dans la guerre et la diplomatie, elle constituait également une association de paix pour ses membres.

6 Le chapitre 5 («Conceptualization of the League ») revient sur les problèmes d'identité et de contours juridiques d'une telle organisation. Ses membres connaissent bien les procédures de représentation dérivées du droit romain. Ainsi, à travers l'étude du vocabulaire de l'association, très riche au XII ${ }^{e}$ siècle (universitas, corpus, societas, civitas, commune, etc.), G. Raccagni essaie de mieux percevoir l'idée que la Ligue se fait d'ellemême ou que les autres s'en font, et piste les évolutions de la Ligue allant d'une simple coniuratio à un véritable corpus. L'étude du vocabulaire, dans les documents d'archives comme dans les sources narratives, permet de réfléchir plus largement sur l'affirmation de l'identité lombarde au cours de ces siècles.

7 L'auteur reprend ensuite une démarche plus chronologique: dans le chapitre 6 tout d'abord (« Reconciliation with the Emperor after the Peace of Constance, 1184-1199»), il évoque, carte à l'appui, les renouvellements de serments d'association prêtés en 1185, 1195 et 1198. En janvier 1185, par exemple, dans l'église Sainte-Brigitte de Plaisance, une assemblée composée des recteurs de douze villes jure de respecter les termes de la paix de Constance : l'association est confirmée pour trente ans, mais le serment pouvait être refait tous les cinq ans si la majorité des recteurs le demandait. Dans ces années, d'autres societates se développent, comme en Toscane ou dans la Marche d'Ancône, dont certaines ont une structure proche de celle de la Ligue lombarde. Dans ces années aussi, le pape Innocent III ne mentionne plus directement la Ligue lombarde dans ses lettres, mais se réfère à plusieurs reprises aux rectores Lombardie. Le chapitre 7 enfin («The Lombard League/Milanese Party, 1200-1225») traque les traces de la Ligue lombarde dans le premier quart du XIII ${ }^{\mathrm{e}}$ siècle. G. Raccagni souligne que la documentation concernant la Ligue diminue de façon drastique à cette époque et qu'aucun témoignage de l'activité de ses recteurs n'est conservé durant cette période, malgré le renouvellement du serment de l'association en 1208 et les traces qu'a laissées la Ligue dans certaines lettres pontificales notamment. À l'inverse, cependant, il n'y a aucune preuve de la disparition de la Ligue. Mais après la mort de Philippe de Souabe, les témoignages relatifs à l'existence de la Ligue deviennent de plus en plus minces. Dans le premier quart $\mathrm{du}$ XIII ${ }^{\mathrm{e}}$ siècle, la Ligue lombarde évolue et se métamorphose, pour l'auteur, en un « parti milanais » (Milanese party). 
8 Ainsi, l'ouvrage de G. Raccagni, qui demeure classique dans sa composition et son approche des sources, offre une synthèse très à jour et très pratique sur ce sujet déjà beaucoup traité par le passé, et montre bien que la Ligue lombarde ne fut pas juste une alliance militaire temporaire, mais bien une association régionale originale et complexe. 